\title{
$\underline{\text { Isolated Uveal Amyloidoma in the absence of Systemic Amyloidosis }}$
}

Guy S Negretti ${ }^{1}$

Caroline Thaung ${ }^{1,2}$

Mandeep S. Sagoo ${ }^{1,2}$

${ }^{1}$ Ocular Oncology Service,

Moorfields Eye Hospital,

London,

EC1V 2PD

United Kingdom

${ }^{2} \mathrm{UCL}$ Institute of Ophthalmology

Bath Street,

London,

EC1V 9EH

United Kingdom

Corresponding author:

Guy Negretti

Moorfields Eye Hospital NHS Foundation Trust,

162 City Road,

London,

EC1V 2PD

United Kingdom

Email: g.negretti@nhs.net

Phone: +442072533411 


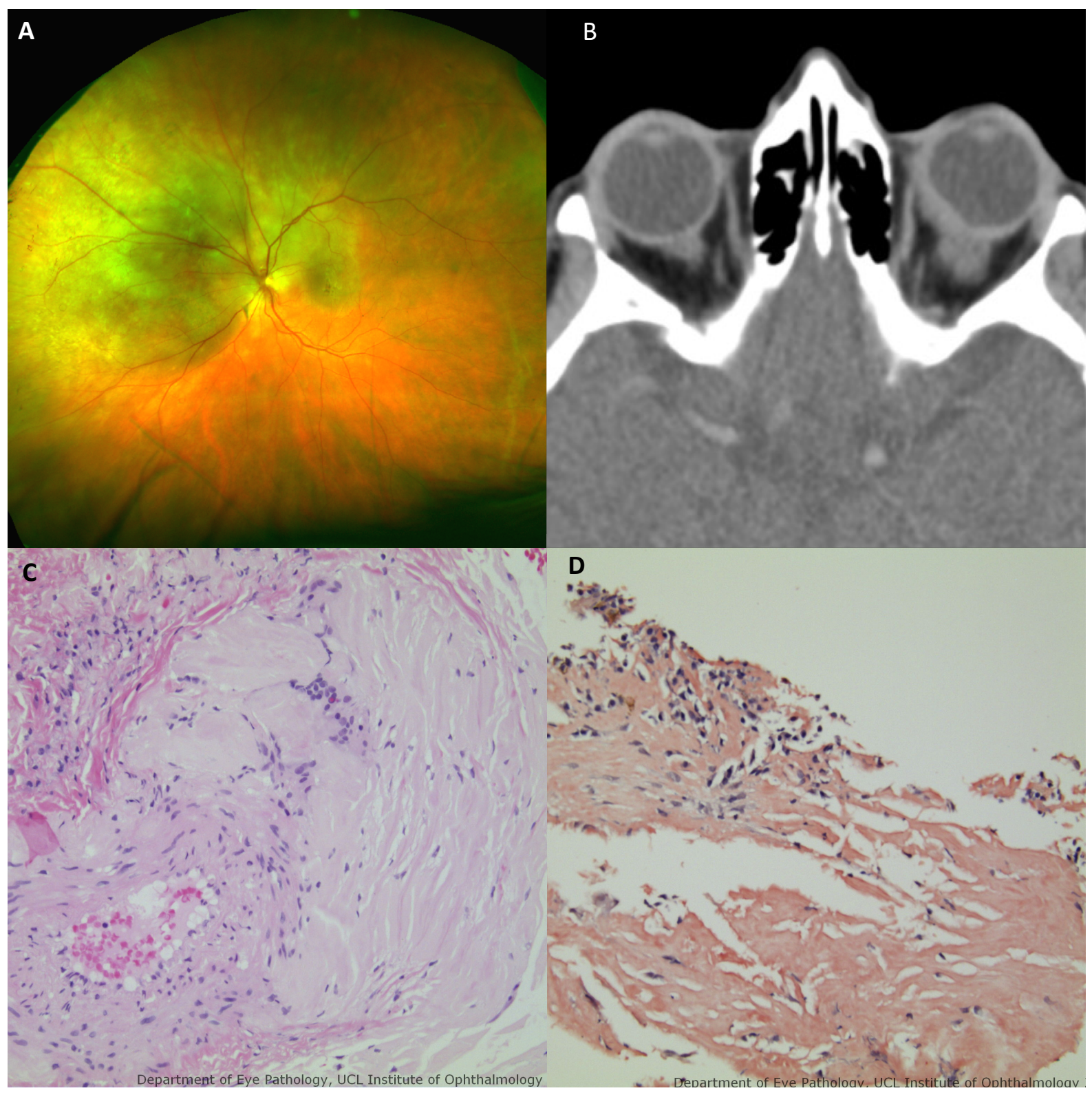

A 70-year-old white woman presented with 2 weeks of left photopsia and scotoma. Clinical examination revealed a choroidal mass without subretinal fluid or lipofuscin (Fig A). B scan showed medium-high echogenicity with extrascleral extension. The latter confirmed on computed tomography scan (Fig B). Orbital biopsy (Fig C) demonstrated amyloid deposits with perivascular accentuation. Systemic evaluation for visceral amyloid or blood dyscrasia was negative. Choroidal lymphoma was suspected, but choroidal biopsy also demonstrated 
amyloid (Fig D, Congo Red) with no evidence of lymphoma. This is a unique case of isolated uveal amyloidoma. The mass stabilised with external beam radiotherapy. 